\title{
CRISPR/Cas9 inhibits rather than induces non-targeted DNA cleavage more likely to cause off-target single-nucleotide variants
}

\author{
Ze Zhang ${ }^{1}$, Yuanyuan Guo ${ }^{1}$, Rongjia Zhang ${ }^{1}$, Wuchen Yang $^{1}$, Zhengqing Xie ${ }^{1}$, Weiwei \\ Chen $^{1}$, Hua Ling ${ }^{1}$, Gangqiang $\mathrm{Cao}^{1}$, Baoming Tian ${ }^{1}$, Fang $\mathrm{Wei}^{1}$, and shi gongyao ${ }^{1}$ \\ ${ }^{1}$ Zhengzhou University
}

September 25, 2021

\begin{abstract}
CRISPR/Cas9 gene targeting technology has become the most widely used gene editing technology in both plants and animals. However, substantial off-target effect remains as a major imperfection hindering its further application. Here, Nicotiana benthamiana leaf cell-free system was used to simulate in vivo environment. And the effects of different CRISPR/Cas9 components on DNA stability in cell-free system were studied to explore possible mechanisms causing CRISPR off-target. The results showed that overexpressing Cas9, nCas9 and dCas9 significantly inhibited DNA cleavage in the cell extracts. While overexpressing RNPs accelerated the target DNA cleavage but inhibited non-target DNA digestion in cell extracts, overexpressing nRNP and dRNP blocked the cleavage of either target or non-target sequences. Meanwhile, analysis of whole-genome sequencing data from mice and rice edited by different CRISPR tools revealed that the main off-target mutations were SNVs (single nucleotide variants), rather than Indels (insertions and deletions) that were readily induced by DNA double-strand breaks. The off-target sites did not match the conventionally predicted places but were PAM-rich sites preferred. Our study suggests that PAM-dependent binding without cleavage of CRISPR/Cas9 to non-target sequences may increase off-target mutation risks through impeding the necessary cleavage process for repairing spontaneous or environmentally induced non-targeted DNA mutations.
\end{abstract}

\section{Hosted file}

CRISPRCas9 inhibits rather than induces non-targeted DNA cleavage more likely to cause off-target singl available at https://authorea.com/users/436169/articles/538689-crispr-cas9-inhibitsrather-than-induces-non-targeted-dna-cleavage-more-likely-to-cause-off-target-singlenucleotide-variants 
A
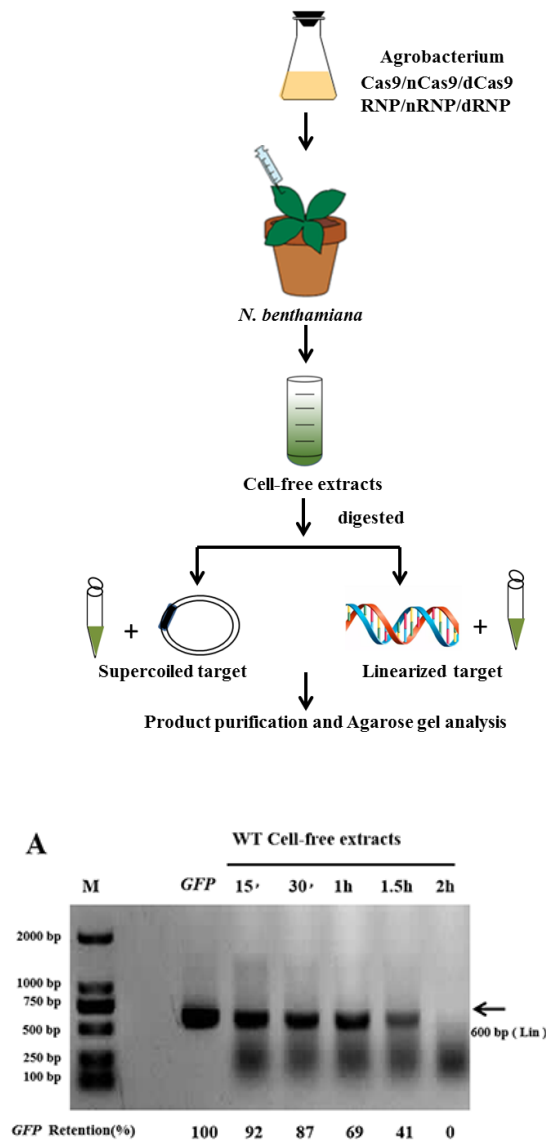

C

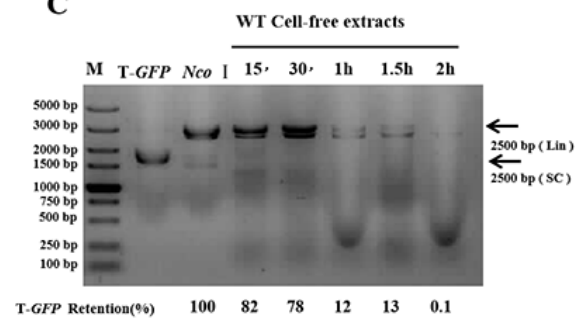

B
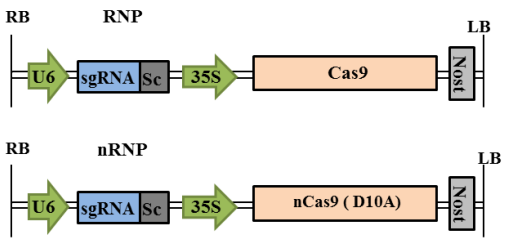

$=\sqrt{\mathrm{U} 6}=\mathrm{sgRNA}_{\mathrm{Sc}}^{\mathrm{RB}}=35 \mathrm{~S}=\mathrm{dCas9}(\mathrm{D10A}, \mathrm{H804A})$

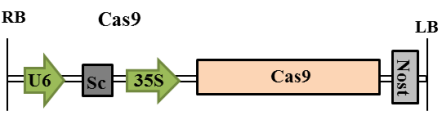

$=\sqrt{\mathrm{U} 6}=\mathrm{Sc}=35 \mathrm{~S}=\mathrm{nCas9}$ ( D10A)
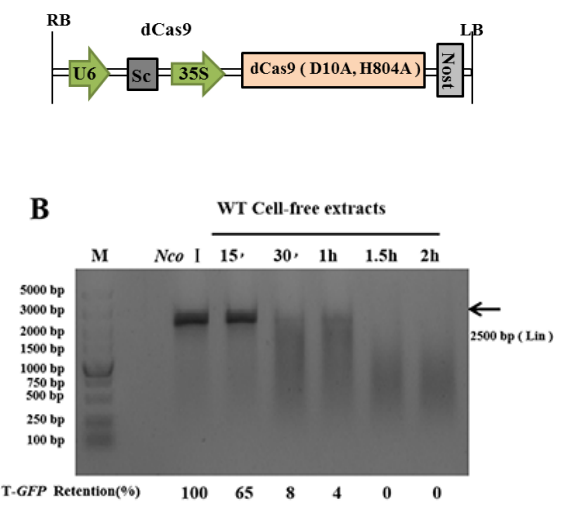

D

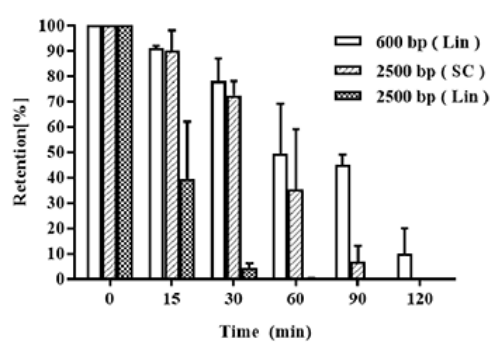


A

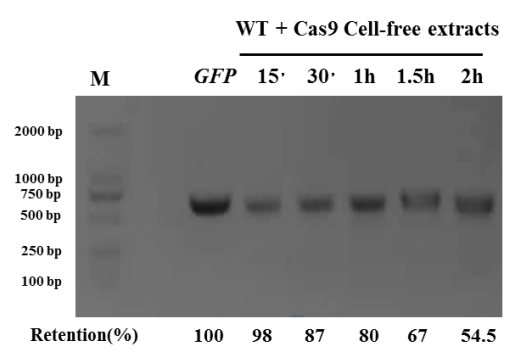

B

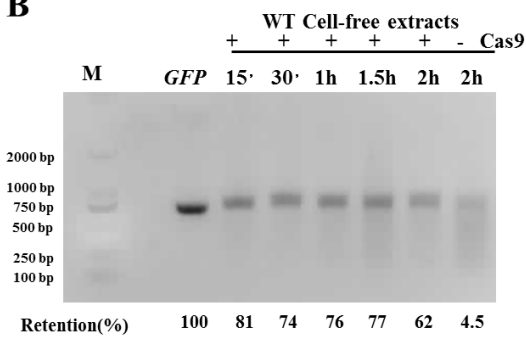

C
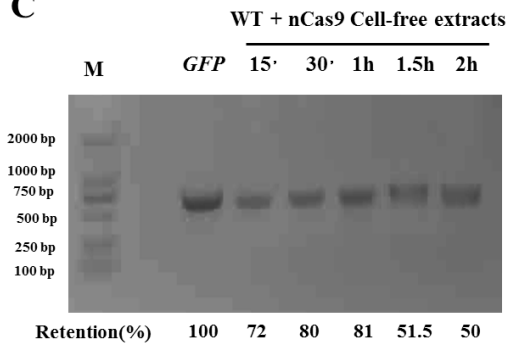

D

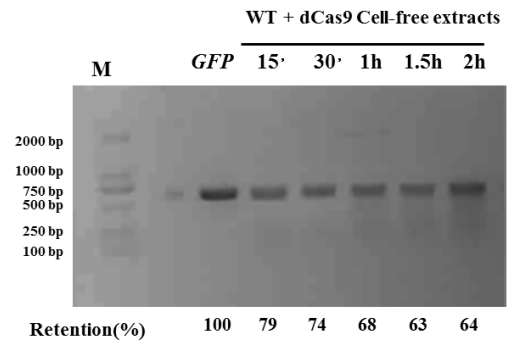

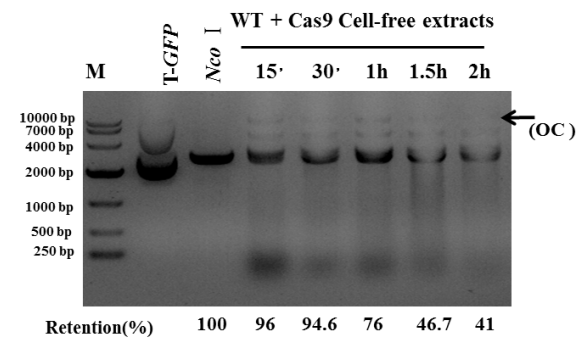
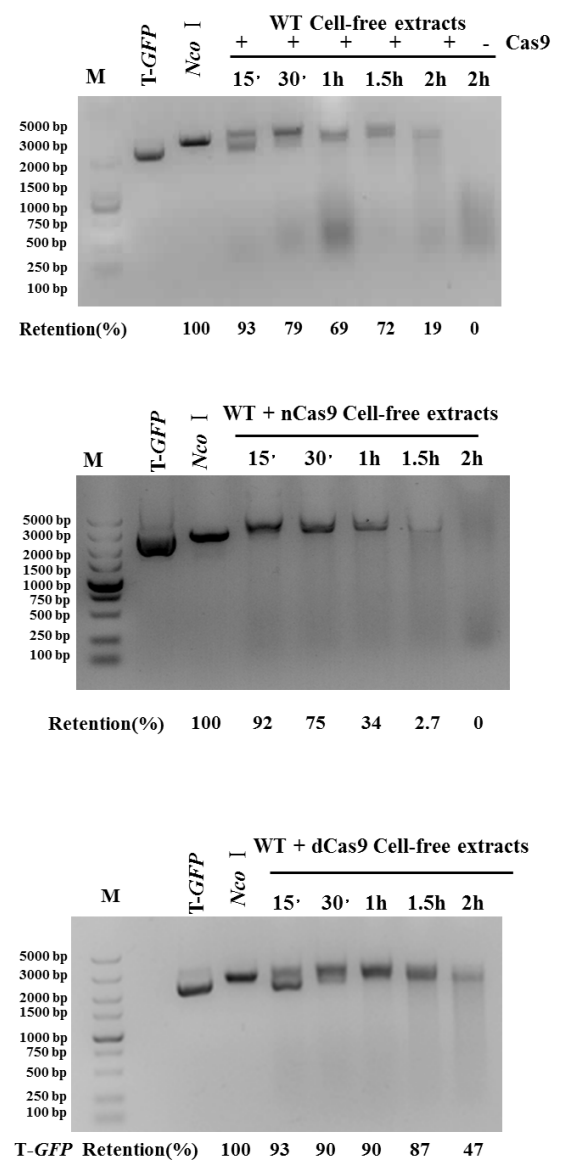
A

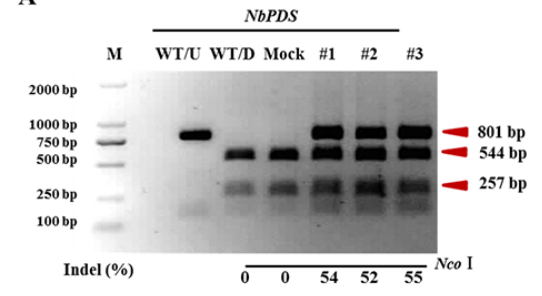

B

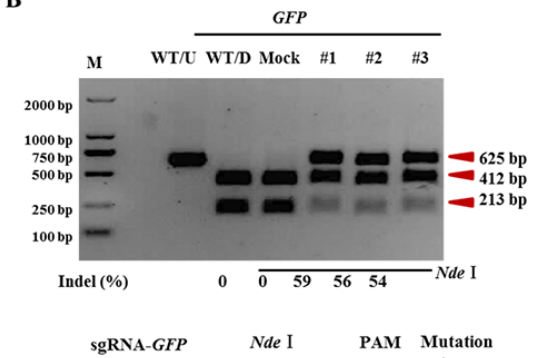

WT GATACCCAGATCATATGAAGCG type

$01(1 / 4)$ GATACCC--------AAGCGG -10

$02(2 / 4)$ GATACCCAGAT------AAGCGG -6

03 (1/4) GATACCCAGATCA----AAGCGG -4

D $\begin{gathered}\text { WT + RNP (sgGFP) } \\ \text { Cell-free extracts } 16 \mathrm{C}\end{gathered}$

M GFP \begin{tabular}{llllll} 
& \multicolumn{4}{c}{ Cell-free extracts $16 \mathrm{C}$} \\
\cline { 2 - 5 } & $15 \cdot 30,1 \mathrm{~h}$ & $1.5 \mathrm{~h}$ & $2 \mathrm{~h}$
\end{tabular}

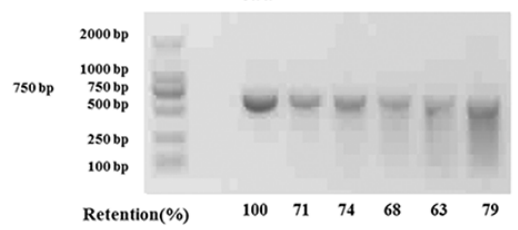


A

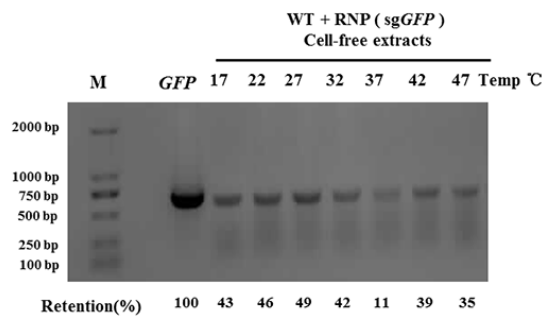

B

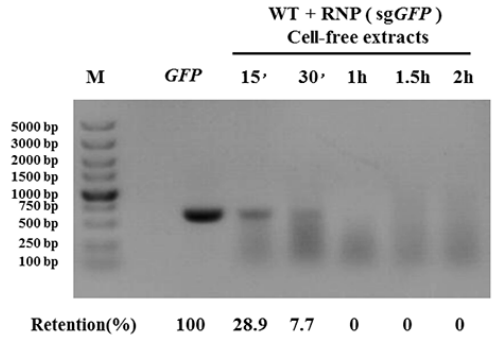

C

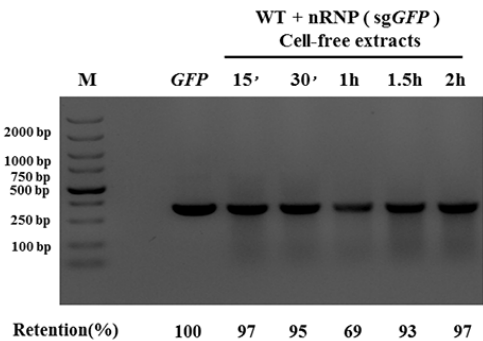

D

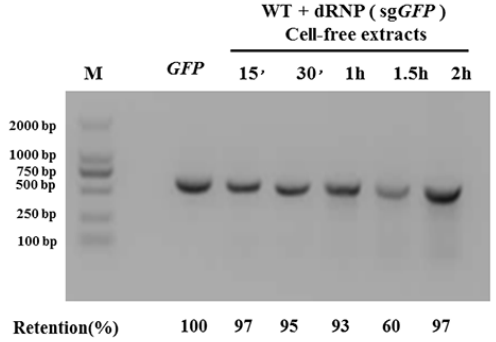

E
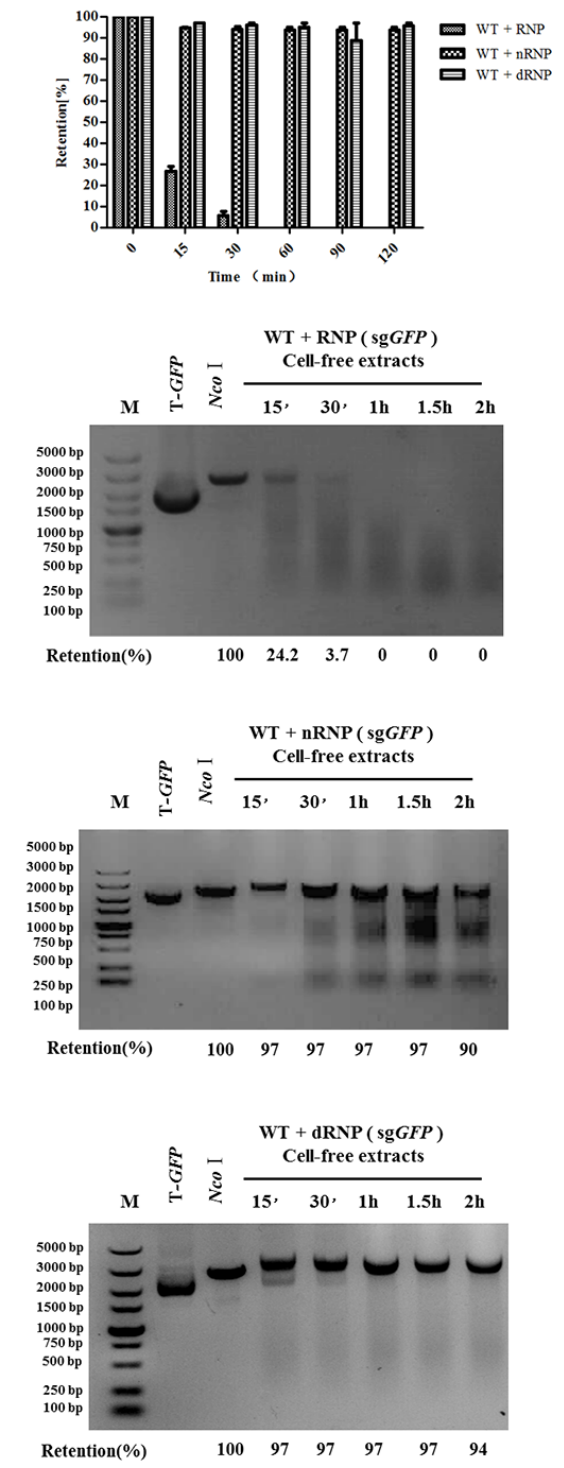
$A_{\text {a } 1}$

RNP extracts targeting double mutants $\mathrm{mGF}$

Ndel PaM
WT GataccCagatchatgaAGCGG

1 GATCACCAGATCAAATGAAGCGG

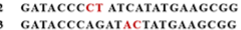

GatACCCAGATCATAGTAAGCGG

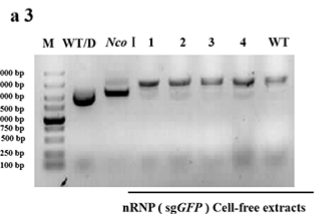

nRNP(sgGFP) Cell-free extracts

C

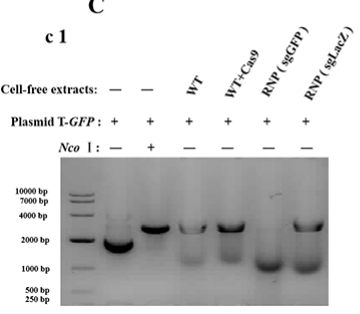

a 2 M WTID NeO $1 \quad 1_{1} \quad 2_{3} \quad 4 \quad$ WT

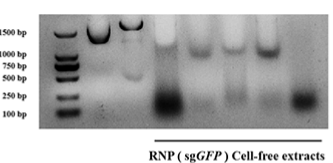

a 4

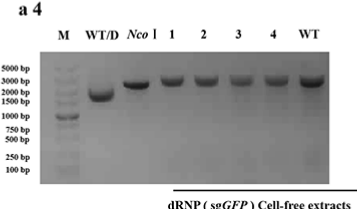

$\overline{\text { dRPP (sGGFP }) \text { Cellfree extracts }}$

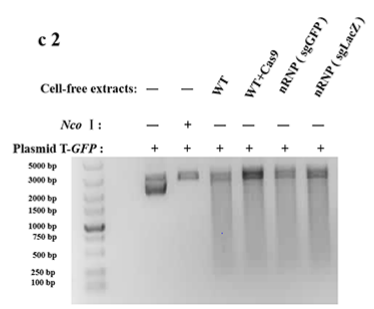

b 1 vxtracts targeting mGFP deletion mutants

Nael PaM CAT AAGCGG 3 GATACCCAGATCA--_AAG-

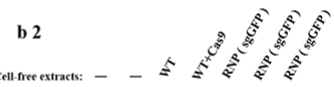

Plasmid: WT WT WT WT $01 \quad 02 \quad 03$

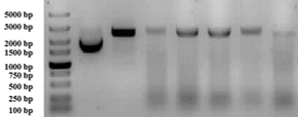

Cell-free extracts: - - 3

Nco I: -+-+-

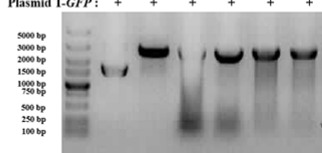

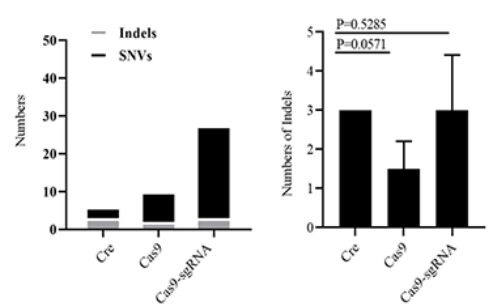

E

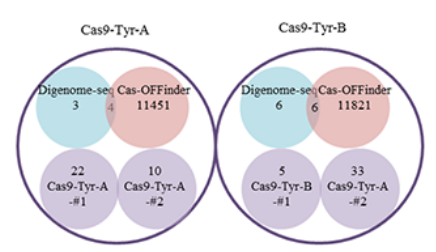

C

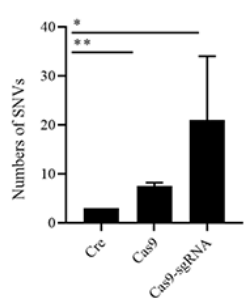

D
F

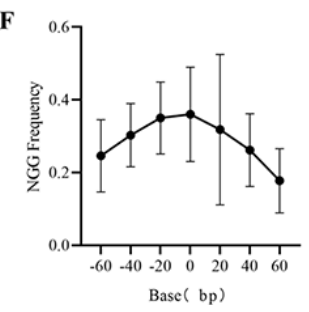


A

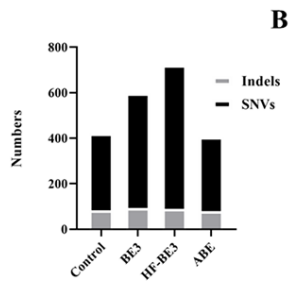

D

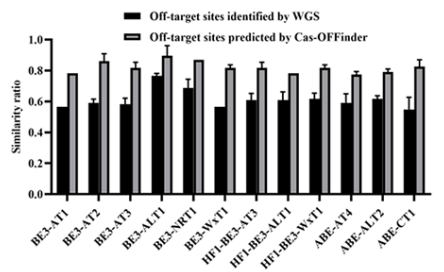

F

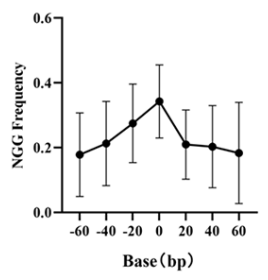

H

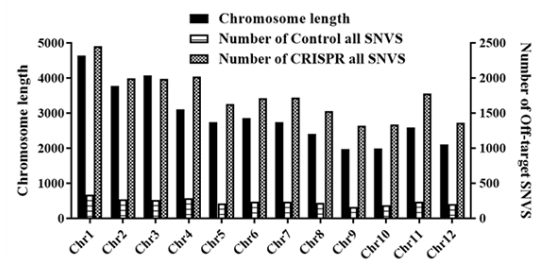

C

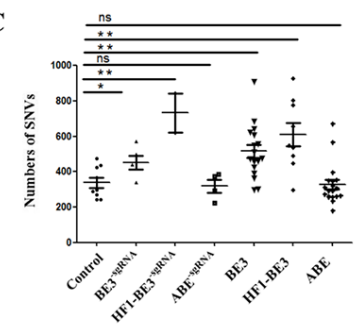

E

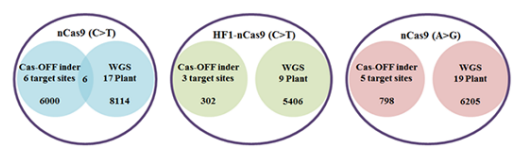

G

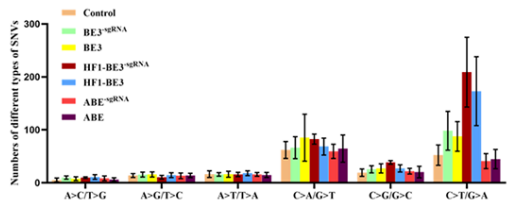

I

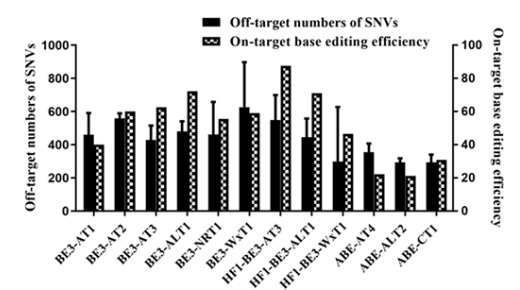

